\title{
Application of the Monte Carlo algorithm for solving volume integral equation in light scattering simulations
}

\author{
MACIEJ KRASZEWSKI, JERZy PLUCIŃSKI* \\ Department of Metrology and Optoelectronics, \\ Faculty of Electronics, Telecommunications and Engineering, \\ Gdańsk University of Technology, Gabriela Narutowicza 11/12, 80-233 Gdańsk, Poland \\ ${ }^{*}$ Corresponding author: pluc@eti.pg.edu.pl
}

\begin{abstract}
Various numerical methods were proposed for analysis of the light scattering phenomenon. An important group of these methods is based on solving the volume integral equation describing the light scattering process. The popular method from this group is the discrete dipole approximation. Discrete dipole approximation uses various numerical algorithms to solve the discretized integral equation. In the recent years, the application of the Monte Carlo algorithm as one of them was proposed. In this research, we analyze the application of the Monte Carlo algorithm for two cases: the light scattering by large particles and by random conglomerates of small particles. We show that if proper preconditioning of the numerical problem is applied, the Monte Carlo algorithm can solve the underlying systems of linear equations. We also show that the efficiency of the Monte Carlo algorithm can be increased by reusing performed computations for various incident electromagnetic waves and the applicability of the Monte Carlo algorithm depends on the particular use case. It is unlikely to be used in the case of light scattering by the large particles due to computational times inferior comparing with the other numerical methods but may become useful in the case of light scattering by the random conglomerates of small scattering particles.
\end{abstract}

Keywords: light scattering, numerical methods, Monte Carlo, discrete dipole approximation (DDA).

\section{Introduction}

The light scattering phenomenon is an important field in both basic and applied research. The large part of this research is the development of numerical methods that would facilitate analysis of light scattering in various cases such as light scattering by single scattering particles with arbitrary shapes and sizes [1-4], scattering by agglomerates of such particles [ $\underline{5}]$, or scattering by macroscopic size random media $[\underline{6}, \underline{7}]$.

Up to date, various numerical approaches to light scattering problems were developed. One of them is direct solving of Maxwell's equations by such methods as finite -difference time-domain (FDTD) or finite element algorithms [ $\underline{8}, \underline{9}]$. Other approach is to use the mode-matching method similar to the classical method for solving light 
scattering problem on arbitrary sized spherical particles developed by Mie $[\underline{2}, \underline{10}]$. This approach allows us to simulate the light scattering process by both single particles as well as by the particle aggregates [2].

The research presented in this paper is focused on the approach based on the so-called volume integral equation (VIE) for light scattering simulations. This method consists in transforming the Helmholtz differential equation into the integral form using the Green's function formalism $[\underline{2}, \underline{10}, \underline{11}$. Probably the most popular version of the VIE approach is the discrete dipole approximation (DDA) that was successfully applied to scattering of light by single particles with arbitrary shapes and sizes [1]. The VIE itself is not a numerical method but only the equation describing the light scattering phenomenon that can be solved in more than one way. Usually, the equation is discretized that leads to the system of algebraic equations. For small problems, these system of equations can be solved directly using the lower-upper (LU) decomposition. Larger problems required iterative methods, e.g. the conjugated gradients or the generalized minimum residual (GMRES) algorithm [1]. One can also exploit the regularity of the discretization grid to accelerate the iterative solvers using the fast Fourier transform (FFT) algorithm for matrix-vector multiplication $[1,12]$.

An interesting approach to solve VIE is the Monte Carlo (MC) algorithm. It is well known that the MC methods can be used to solve Fredholm integral equations like the VIE for light scattering problems. This approach has been studied in different fields of science [13-16]. Also, there are few reported attempts to use the MC algorithm for light scattering simulation but no actual analysis of its performance and usefulness has been provided $[17,18]$. To the best of our knowledge, the topic of using the $\mathrm{MC}$ algorithm to solve VIE in light scattering problems was not deeply analyzed in the scientific literature. In particular, there is lack of research on preconditioning of the numerical problem solved by the MC algorithm, which we show to be crucial for its successful implementation.

At this point, we would like to resolve a possible naming ambiguity. In the light scattering studies, there is another widely used numerical method that also comes with the name of the MC method [ $\underline{6}, \underline{7}]$. This is a popular numerical approach to solve the radiative transfer equation (RTE) describing the propagation of the radiation in the averaged realization of the random scattering medium. This is a different problem than analyzing of the light scattering by the VIE that is discussed in this paper. The RTE is used in the problem of light propagation in the large volume of the averaged realization of the scattering medium and does not directly concern the wave nature of the radiation. On the other hand, the VIE is used for analyzing scattering of light on objects with dimensions in range up to hundreds of micrometers and directly treats the wave nature of the electromagnetic radiation. Further, in this paper we will use the term MC method only for a method of solving the VIE.

The goal of the presented research was to analyze the applicability of the MC method in the light scattering problems. First, we present the mathematical framework for analyzing light scattering using the VIE along with the well-known DDA method. Next, we present the MC algorithm for solving the VIE as well as the convergence criterion 
for this algorithm. We show that in its simplest implementation, the $\mathrm{MC}$ algorithm has a simple physical interpretation of sampling various paths of the electromagnetic wave scattered by small particles. However, without the proper preconditioning of the numerical problem, the MC algorithm fails to converge for many typical light scattering problems. Finally, we compare the MC and other algorithms in terms of speed and accuracy.

We conclude that the MC algorithm offers certain advantages over other methods since it is easy to implement and does not require the storage of large dense matrices in the computer memory. It can also provide a solution of the scattering problem but only with limited accuracy that limits its range of applications. The behavior of the $\mathrm{MC}$ algorithm also depends on its specific implementation and the parameters of the analyzed problem and since that its wide application in studies of the light scattering phenomenon requires further research.

\section{Description of the numerical algorithm}

\subsection{Volume integral equation for light scattering problem}

The VIE for light scattering problem can be derived directly from the Helmholtz equation

$$
\nabla^{2} \mathbf{E}(\mathbf{r})+k_{0}^{2} n^{2}(\mathbf{r}) \mathbf{E}(\mathbf{r})=0
$$

where $\mathbf{r}$ is the position of the point in space, $\mathbf{E}$ is the complex amplitude of the electric field, $k_{0}$ is the wave number in the vacuum, and $n$ is the light refractive index.

Further part of this paper concerns a conglomerate of small scattering particles inside the free space. Therefore, we assume that $n^{2}(\mathbf{r})=1$ everywhere besides the interior of the particles. form

Using the Green's function formalism, Eq. (1) can be transformed into the integral

$$
\mathbf{E}(\mathbf{r})=\mathbf{E}^{\mathrm{inc}}(\mathbf{r})+\int_{V}^{\overleftrightarrow{\mathbf{G}}}\left(\mathbf{r}, \mathbf{r}^{\prime}\right) U\left(\mathbf{r}^{\prime}\right) \mathbf{E}\left(\mathbf{r}^{\prime}\right) \mathrm{d}^{3} \mathbf{r}^{\prime}
$$

where $\mathbf{E}^{\text {inc }}(\mathbf{r})$ is the electric field incident onto the particles (e.g. from an external light source), $\overleftrightarrow{\mathbf{G}}\left(\mathbf{r}, \mathbf{r}^{\prime}\right)$ is the dyadic Green's function, $U\left(\mathbf{r}^{\prime}\right)$ is the so-called scattering potential, $V$ is volume, and the integral in Eq. (2) is evaluated over the entire space.

The dyadic Green's function is given by equation

$$
\overleftrightarrow{\mathbf{G}}\left(\mathbf{r}, \mathbf{r}^{\prime}\right)=\left(\overleftrightarrow{\mathbf{I}}-\frac{1}{k_{0}^{2}} \nabla \otimes \nabla\right) \frac{\exp \left(i k_{0}\left|\mathbf{r}-\mathbf{r}^{\prime}\right|\right)}{4 \pi\left|\mathbf{r}-\mathbf{r}^{\prime}\right|}
$$

where $\overleftrightarrow{\mathbf{I}}$ is the identity dyadic and $\mathbf{a} \otimes \mathbf{b}$ is a dyadic defined by two vectors $\mathbf{a}$ and $\mathbf{b}$ $[\underline{2}, \underline{11}]$.

The scattering potential is equal to:

$$
U(\mathbf{r})=k_{0}^{2}\left[n^{2}(\mathbf{r})-1\right]
$$


The detailed derivation and discussion of Eq. (2) can be found in numerous textbooks on optics and light scattering $[\underline{2}, \underline{11}]$. Here we just point out a few of its features. First, Eq. (2) divides the total electric field of an electromagnetic wave $\mathbf{E}(\mathbf{r})$ into the incident field and the scattered field:

$$
\mathbf{E}(\mathbf{r})=\mathbf{E}^{\text {inc }}(\mathbf{r})+\mathbf{E}^{\mathrm{sca}}(\mathbf{r})
$$

where $\mathbf{E}^{\mathrm{inc}}(\mathbf{r})$ is the incident field and $\mathbf{E}^{\text {sca }}(\mathbf{r})$ is the scattered field equal to

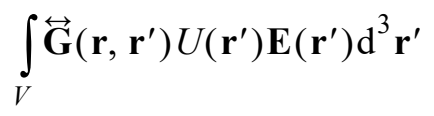

Secondly, the scattering potential $U(\mathbf{r})=0$ everywhere outside the scattering particles, so the integral in Eq. (2) need to be evaluated only for the volume inside the particles.

\subsection{Discretization of the equations}

Discretization of Eq. (2) is consists in the division of the volume $V$ for $N$ non-overlapping subvolumes (particles). It is assumed that the particles are small enough, so that the electric field and scattering potential inside them are constant.

Integration of Eq. (2) for all particles leads to the following equation:

$$
\mathbf{E}_{i}=\mathbf{E}_{i}^{\mathrm{inc}}+\sum_{j \neq i} V_{j} U_{j} \overleftrightarrow{\mathbf{G}}_{i j} \mathbf{E}_{j}+U_{i}\left(\overleftrightarrow{\mathbf{M}}_{i}-\overleftrightarrow{\mathbf{L}}_{i}\right) \mathbf{E}_{i}
$$

where $\mathbf{E}_{i}, \mathbf{E}_{i}^{\mathrm{inc}}, U_{i}$, and $V_{i}$ are the total electric field, the incident electric field, the scattering potential, and the volume of the $i$-th particle, respectively, $\overleftrightarrow{\mathbf{G}}_{i j}$ is the dyadic Green's function for $\mathbf{r}=\mathbf{r}_{i}$ being the position of the $i$-th particle and $\mathbf{r}^{\prime}=\mathbf{r}_{j}$ being the position of the $j$-th particle.

Exclusion of index $j=i$ from the sum in Eq. (6) and addition of term $U_{i}\left(\overleftrightarrow{\mathbf{M}}_{i}-\overleftrightarrow{\mathbf{L}}_{i}\right) \mathbf{E}_{i}$ comes from the fact that the dyadic Green's function has a singularity for $\mathbf{r}=\mathbf{r}^{\prime}$ and this case needs to be treated separately. A more detailed discussion of this problem is out of the scope of this paper and can be found, e.g. in works $[1,19]$.

We further follow the DDA approach presented in []] where Eq. (6) is being solved not for the total electric field but for the total polarizations of the particles

$$
\mathbf{P}_{i}=V_{i} U_{i} \mathbf{E}_{i}=\alpha_{i} \mathbf{E}_{i}
$$

what leads to the following equation [1]:

$$
\mathbf{P}_{i}=\overleftrightarrow{\boldsymbol{\alpha}}_{i} \mathbf{E}_{i}^{\mathrm{inc}}+\overleftrightarrow{\boldsymbol{\alpha}}_{i} \sum_{j \neq i} \overleftrightarrow{\mathbf{G}}_{i j} \mathbf{P}_{j}
$$

for

$$
\overleftrightarrow{\boldsymbol{\alpha}}_{i}=V_{i} U_{i}\left(\overleftrightarrow{\mathbf{I}}+\left(\overleftrightarrow{\mathbf{L}}_{i}-\overleftrightarrow{\mathbf{M}}_{i}\right) U_{i}\right)^{-1}
$$


By collecting equations for polarizations of all particles, one can obtain the system of linear equations that can be presented in the standard form

$$
\mathbf{A x}=\mathbf{B}
$$

for

$$
\mathbf{x}=\left[\begin{array}{c}
\mathbf{P}_{1} \\
\vdots \\
\mathbf{P}_{N}
\end{array}\right]
$$

and

$$
\mathbf{b}=\left[\begin{array}{c}
\mathbf{E}_{1}^{\text {inc }} \\
\vdots \\
\mathbf{E}_{N}^{\text {inc }}
\end{array}\right]
$$

The MC algorithm is based on Eq. (10) transformed into the different form

$$
\mathbf{x}=\mathbf{b}+\mathbf{G x}
$$

$$
\text { for } \mathbf{G}=\mathbf{I}-\mathbf{A} \text {. }
$$

In further part of this paper, we apply the above mathematical formalism to the two problems. The first problem concerns light scattering by a large spherical particle (Fig. 1a). In this case, the entire particle is divided into the numerous small subparticles (usually called as dipoles) for which Eq. (8) is solved. The dipoles are tightly packed and fill the entire particle volume. The second problem concerns the scattering of light by a conglomerate of sparsely distributed scattering particles (Fig. 1b). We assume that

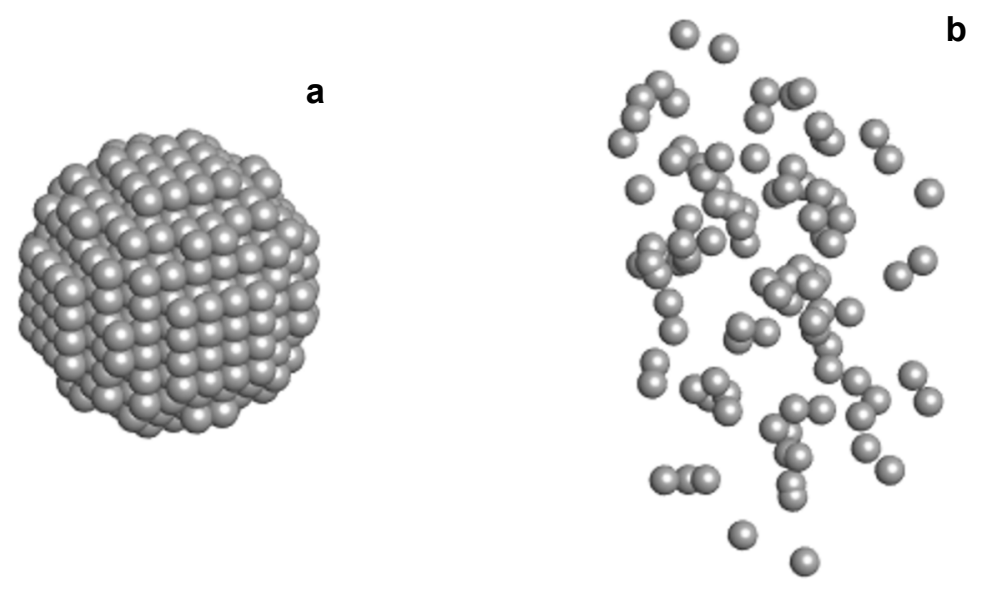

Fig. 1. Geometry of analyzed light scattering problems: light scattering by a large spherical particle (a), and light scattering by a random conglomerate of small particles (b). 
particles are small enough, so that each of them can be represented by a single dipole. We show a comparison of solving Eq. (8) for both types of problems using the GMRES algorithm and $\mathrm{MC}$ algorithms.

\subsection{Monte Carlo algorithm for solving the system of linear equation}

The system of linear equations in the form given by Eq. (10) can be solved using the well-known MC algorithm [13-18]. This approach is based on the Neumann expansion of Eq. (13):

$$
\mathbf{x}=\mathbf{b}+\mathbf{G b}+\mathbf{G}^{2} \mathbf{b}+\mathbf{G}^{3} \mathbf{b}+\ldots
$$

By using the $\mathrm{MC}$ algorithm, one can obtain a random estimator of the solution to Eq. (14) by the random sampling of the trajectories of a Markov chain process. Here we describe the $\mathrm{MC}$ algorithm using the analogy to sampling paths of photons scattered by small particles.

The first term $\mathbf{b}$ in the right-hand side of Eq. (14) is simply a polarization of particles caused by the incident electromagnetic wave. The second term $\mathbf{G b}$ can be interpreted as a polarization of particles caused by the incident wave scattered only once by each of the scattering particles. The third term $\mathbf{G}^{2} \mathbf{b}$ can be interpreted as the polarization caused by the wave scattered twice, etc. While this analogy does not fully represent the physical process of light scattering [2] , it is useful for understanding the principles of the $\mathrm{MC}$ algorithm. Hence, it is possible to think of Eq. (14) as of the sum of polarization caused by the wave scattered multiple times between the particles and of the MC algorithm as of the random sampling of the wave trajectories. Instead of summing all possible wave trajectories, we consider only a randomly selected finite subset of them.

We define the transition matrix $\mathbf{T}$ as a matrix of probabilities that fulfills the following conditions:

$$
\begin{aligned}
& g_{i, j} \neq 0 \Rightarrow t_{i, j}>0 \\
& \forall_{j} \sum_{i=1}^{M} t_{i, j}=1-t_{j, M+1} \\
& t_{M+1, i}= \begin{cases}1 & \text { for } i=M+1 \\
0 & \text { otherwise }\end{cases}
\end{aligned}
$$

where $g_{i, j}$ and $t_{i, j}$ denote elements of the matrices $\mathbf{G}$ and $\mathbf{T}$, respectively, and $M$ is the dimension of the matrix $\mathbf{G}$. We will denote $t_{i, M+1}$ as the termination probability.

To estimate the solution vector $\mathbf{x}$, we generate the Markov chain (trajectories) from the Markov process with $M+1$ states. The transition probabilities between states are given by the matrix T. From Eq. (17), it is clear that the state $M+1$ is the absorbing state. We will further denote the set of states of the generated trajectory with length $K$ as $\left\{i_{0}, \ldots, i_{K-1}\right\}$. We also denote the probability of the initial state being $i_{0}$ to $\alpha_{i_{0}}$. 
For every trajectory we build the estimator to the vector $\mathbf{x}$ according to equation

$$
\tilde{x}_{j}=\frac{\delta_{j, i_{K-1}}}{t_{i_{K-1}, M+1}} \frac{g_{i_{K-1}, i_{K-2}}}{g_{i_{K-2}, i_{K-1}}} \ldots \frac{g_{i_{1}, i_{0}}}{t_{i_{0}, i_{1}}} \frac{b_{i_{0}}}{\alpha_{i_{0}}}
$$

where $\tilde{x}_{j}$ is the $j$-th component of the estimated solution to Eq. (14), $b_{i}$ is the $i$-th component of the vector $\mathbf{b}$, and $\delta_{i, j}$ is the Kronecker delta. Note that a single trajectory is used to estimate only one entry of the vector $\mathbf{x}$.

The final estimation of the vector $\mathbf{x}$ is given by the average of numerous estimators given by Eq. (18). It is known that this estimator is unbiased [13].

\subsection{Modifications of the Monte Carlo algorithm}

In this paper, we propose the usage of the modified version of the MC algorithm described in the previous section. First, we generate only trajectories with the length not exceeding a certain threshold. This introduces the bias to the estimator from Eq. (18) but reduces its variance. Therefore, provided that the convergence criterion is met, long trajectories have a decreasing impact on the overall result.

Second, we reuse generated trajectories to obtain the estimation of all entries of the solution vector $\mathbf{x}$. This modification uses the fact that all terms in the product from the right-hand side of Eq. (18), except for the two first of them, repeat in estimators of different entries of the solution vector.

Finally, we deterministically include all trajectories with only one state. This can be interpreted as approximating the total electric field on the scattering particles as being equal to the incident electric field and the usage of the $\mathrm{MC}$ algorithm only to include a higher-order term in the Neumann expansion.

It is also possible to reuse the generated trajectories to simulate the solution for various vectors $\mathbf{b}$. This can be useful if simulations need to be repeated for the same scattering object but for different parameters of the incident wave, e.g. the polarization, the propagation direction etc. In Section 2.3, we show computational speed-up that can be obtained using this technique.

\subsection{Motivation for using the Monte Carlo algorithm}

It is known that the MC algorithm described in Section 2.4. requires a lot of computational time in order to provide high accuracy solution to the underlying system of equations. However, it has certain advantages that make it worth exploring [13]:

- it can quickly provide an approximate solution to the analyzed problem,

- it allows us to solve simultaneously for multiple vectors $\mathbf{b}$,

- it allows us to find a solution only for a particular element of the desired solution,

- it is easy to implement and does not require large amounts of computer memory,

- it is easy to parallelize using processors clusters.

In Section 2.4, we discuss if and how these advantages can be exploited in the field of light scattering simulations. 


\subsection{Convergence and preconditioning of the Monte Carlo algorithm}

The MC algorithm converges if the Neumann series from Eq. (14) is convergent, which is true if the spectral radius of matrix $\mathbf{G}$ is smaller than one $[\underline{13}, \underline{15}]$ :

$$
\rho(\mathbf{G})<1
$$

In the further part of this paper, we show that for typical scattering problems solved with the VIE approach, the condition (19) is not fulfilled. For this reason, it is impossible to use the MC algorithm directly on Eq. (13).

The way to deal with the convergence properties of the $\mathrm{MC}$ algorithm is the proper preconditioning of the numerical problem. This means transforming the system of linear equations given by Eq. (10) into the system having the same solution but for which the convergence condition of the MC algorithm is not violated. In this research, we used the so-called left diagonal preconditioning which consists in splitting the matrix $\mathbf{A}$ into its diagonal and non-diagonal parts [르]:

$$
\mathbf{A}=\mathbf{D}-\mathbf{E}
$$

where $\mathbf{D}$ and $\mathbf{E}$ are a diagonal and a non-diagonal part of matrix $\mathbf{A}$, respectively.

Substituting Eq. (20) into (10) gives

$$
\mathbf{D} \mathbf{x}-\mathbf{E x}=\mathbf{b}
$$

and

$$
\mathbf{x}=\mathbf{D}^{-1} \mathbf{b}+\mathbf{D}^{-1} \mathbf{E x}
$$

For $\mathbf{b}^{\prime}=\mathbf{D}^{-1} \mathbf{b}$ and $\mathbf{G}^{\prime}=\mathbf{D}^{-1} \mathbf{E}$, Eq. (22) can be transformed into the same form as Eq. (13):

$$
\mathbf{x}=\mathbf{b}^{\prime}+\mathbf{G}^{\prime} \mathbf{x}
$$

Since $\mathbf{D}$ is a diagonal matrix, both $\mathbf{b}^{\prime}$ and $\mathbf{G}^{\prime}$ can be find in a short time and Eq. (23) can be solved using the MC algorithm. In Section 2.3 we show that this approach indeed allows us to use the MC algorithm for analyzing the light scattering problems.

\section{Numerical simulations}

\subsection{Scattering by a large spherical particle}

We tested the MC algorithm by using the DDA method to compute the scattering cross -section of the spherical particles with the refractive index 1.33 and the diameters varying from $0.25 \lambda$ to $1 \lambda$, where $\lambda$ is the wavelength of the light in the vacuum. The number of dipoles into which the sphere was divided was varying from 32 to 912 . The matrices from Eq. (10) were generated in MATLAB environment using the open-source DDA Toolbox [21] that implements the DDA method described in []ㅡ. The scattering cross -section was computed using the GMRES and MC algorithms. The residual error for 
the GMRES algorithm did not exceed $10^{-5}$. The number of iterations in the MC algorithm was varying from $10^{2}$ to $10^{4}$. The length of the trajectories in the MC algorithm was limited to three what corresponds to estimating first three terms in Neumann expansion. The MC algorithm was implemented in high level programming language - C\#. The computations were performed on Intel i7-6700 HQ CPU operating with $2.4 \mathrm{GHz}$ clock and 32 GB of RAM.

For comparison, the scattering cross-sections were also estimated by direct computing of the Neumann expansion limited to one, two, and three terms. For both MC and direct estimations of Neumann expansion, the preconditioning described in Section 2.6 was used. Since, for the case of direct computation, this preconditioning is the same as in the Jacobi iterative scheme for solving the linear equations, the direct computations are further denoted as Jacobi iterations.

To check the convergence criterion and effectiveness of the applied preconditioning, a spectral radius $\rho(\mathbf{G})$ of the system without preconditioning and $\rho\left(\mathbf{G}^{\prime}\right)$ of the system with preconditioning were computed. For every case, values of $\rho(\mathbf{G})$ exceeded $10^{4}$ that makes the MC and Jacobi iterations divergent without the preconditioning. Values of $\rho\left(\mathbf{G}^{\prime}\right)$ are presented in Table 1 . The results show that for most of the analyzed cases the convergence criterions were met.

The results of computation are presented in Table 2. Accuracies were defined as the relative errors of the computed scattering cross-sections compared to the results obtained with the GMRES algorithm. The calculated cross-sections were compared to the GMRES results and not to the analytical solution obtained with the Mie theory,

$\mathrm{T}$ a b $1 \mathrm{e}$ 1. Effect of preconditioning on solving the discretized VIE for light scattering by the spherical particle with refractive index 1.33.

\begin{tabular}{lll}
\hline Number of dipoles & Sphere diameter $[\lambda]$ & $\rho\left(\mathbf{G}^{\prime}\right)$ \\
\hline 32 & 0.25 & 0.62 \\
32 & 0.5 & 0.64 \\
32 & 0.75 & 0.67 \\
32 & 1.0 & 0.72 \\
280 & 0.25 & 0.75 \\
280 & 0.5 & 0.77 \\
280 & 0.75 & 0.87 \\
280 & 1.0 & 1.31 \\
552 & 0.25 & 0.77 \\
552 & 0.5 & 0.79 \\
552 & 0.75 & 1.20 \\
552 & 1.0 & 1.75 \\
912 & 0.25 & 0.78 \\
912 & 0.5 & 0,87 \\
912 & 0.75 & 1.50 \\
912 & 1.0 & 2.02 \\
\hline
\end{tabular}


$\mathrm{T}$ a b $1 \mathrm{e} 2$. Accuracy of the Monte Carlo simulation of light scattering by spherical particles with refractive index 1.33 .

\begin{tabular}{llrrrrrr}
\hline \multirow{2}{*}{$\begin{array}{l}\text { Number } \\
\text { of dipoles }\end{array}$} & $\begin{array}{l}\text { Sphere } \\
\text { diameter }[\lambda]\end{array}$ & \multicolumn{3}{c}{ Monte Carlo simulation accuracy } & \multicolumn{3}{c}{ Jacobi iteration accuracy } \\
\cline { 2 - 7 } & $10^{2}$ it. & $10^{3}$ it. & $10^{4}$ it. & 1 it. & 2 it. & 3 it. \\
\hline 32 & 0.25 & $8 \%$ & $5 \%$ & $1 \%$ & $4 \%$ & $1 \%$ & $0 \%$ \\
32 & 0.5 & $15 \%$ & $5 \%$ & $1 \%$ & $12 \%$ & $2 \%$ & $0 \%$ \\
32 & 0.75 & $1 \%$ & $3 \%$ & $2 \%$ & $26 \%$ & $5 \%$ & $1 \%$ \\
32 & 1.0 & $29 \%$ & $14 \%$ & $3 \%$ & $40 \%$ & $9 \%$ & $2 \%$ \\
280 & 0.25 & $36 \%$ & $13 \%$ & $5 \%$ & $14 \%$ & $2 \%$ & $0 \%$ \\
280 & 0.5 & $14 \%$ & $31 \%$ & $6 \%$ & $46 \%$ & $11 \%$ & $2 \%$ \\
280 & 0.75 & $40 \%$ & $50 \%$ & $40 \%$ & $87 \%$ & $34 \%$ & $17 \%$ \\
280 & 1.0 & $90 \%$ & $56 \%$ & $33 \%$ & $107 \%$ & $46 \%$ & $27 \%$ \\
552 & 0.25 & $9 \%$ & $10 \%$ & $3 \%$ & $21 \%$ & $4 \%$ & $1 \%$ \\
552 & 0.5 & $23 \%$ & $48 \%$ & $15 \%$ & $67 \%$ & $19 \%$ & $5 \%$ \\
552 & 0.75 & $98 \%$ & $80 \%$ & $21 \%$ & $97 \%$ & $41 \%$ & $25 \%$ \\
552 & 1.0 & $78 \%$ & $70 \%$ & $69 \%$ & $118 \%$ & $64 \%$ & $49 \%$ \\
912 & 0.25 & $10 \%$ & $25 \%$ & $15 \%$ & $29 \%$ & $6 \%$ & $1 \%$ \\
912 & 0.5 & $61 \%$ & $26 \%$ & $31 \%$ & $89 \%$ & $35 \%$ & $18 \%$ \\
912 & 0.75 & $119 \%$ & $130 \%$ & $62 \%$ & $119 \%$ & $62 \%$ & $43 \%$ \\
912 & 1.0 & $91 \%$ & $107 \%$ & $65 \%$ & $127 \%$ & $80 \%$ & $66 \%$ \\
\hline
\end{tabular}

$\mathrm{T} \mathrm{a} \mathrm{b} 1 \mathrm{e}$ 3. Time of the Monte Carlo simulation of light scattering by a spherical particle with refractive index 1.33 .

\begin{tabular}{llcccccc}
\hline \multirow{2}{*}{$\begin{array}{l}\text { Number } \\
\text { of dipoles }\end{array}$} & $\begin{array}{l}\text { Sphere } \\
\text { diameter }[\lambda]\end{array}$ & \multicolumn{3}{c}{ Monte Carlo simulation time [ms] } & \multicolumn{3}{c}{ Jacobi iteration time [ms] } \\
\cline { 2 - 7 } & $10^{2}$ it. & $10^{3}$ it. & $10^{4}$ it. & 1 it. & 2 it. & 3 it. \\
\hline 32 & 0.25 & 0.38 & 1.91 & 18.9 & 0.03 & 0.03 & 0.03 \\
32 & 0.5 & 0.38 & 2.03 & 18.30 & 0.02 & 0.02 & 0.03 \\
32 & 0.75 & 0.32 & 2.68 & 18.60 & 0.001 & 0.03 & 0.05 \\
32 & 1.0 & 0.38 & 2.28 & 20.60 & 0.02 & 0.01 & 0.03 \\
280 & 0.25 & 3.69 & 34.43 & 341.80 & 0.001 & 0.32 & 0.66 \\
280 & 0.5 & 3.98 & 33.77 & 322.30 & 0.002 & 0.30 & 0.61 \\
280 & 0.75 & 4.12 & 33.69 & 322.20 & 0.002 & 0.29 & 0.49 \\
280 & 1.0 & 4.17 & 33.06 & 321.60 & 0.002 & 0.41 & 0.92 \\
552 & 0.25 & 7.9 & 77.55 & 774.70 & 0.01 & 2.24 & 4.00 \\
552 & 0.5 & 11.54 & 88.17 & 946.10 & 0.002 & 2.19 & 4.14 \\
552 & 0.75 & 9.43 & 83.94 & 894.50 & 0.002 & 2.13 & 4.04 \\
552 & 1.0 & 7.64 & 80.07 & 769.60 & 0.002 & 2.18 & 3.86 \\
912 & 0.25 & 18.07 & 169.61 & 1688.10 & 0.002 & 6.62 & 11.82 \\
912 & 0.5 & 13.50 & 132.20 & 1347.80 & 0.002 & 6.37 & 11.22 \\
912 & 0.75 & 17.74 & 160.23 & 1758.70 & 0.002 & 6.06 & 12.42 \\
912 & 1.0 & 13.50 & 130.95 & 1316.90 & 0.002 & 5.59 & 12.80 \\
\hline
\end{tabular}


since the scope of this research was to validate the MC method of solving the discretized VIE and not to validate the accuracy of the DDA itself. The required computation times are presented in Table 3.

\subsection{Simultaneous computations for multiple incident electromagnetic waves}

In order to estimate the reduction of the computation time by reusing the computed $\mathrm{MC}$ trajectories for different incident electromagnetic waves, we performed the MC computation for the sphere divided into 552 dipoles and the trajectories length limited to three. The trajectories were reused to estimate the solution vector $\mathbf{x}$ for multiple vectors $\mathbf{b}$. The number of $\mathrm{MC}$ iterations was equal to $10^{4}$. The required computation times and computational speed-up are presented in Fig. 2. The computational speed-up was defined as

$$
s(k)=k \frac{t(1)}{t(k)}
$$

where $s$ is the speed-up, $k$ is the number of simultaneously analyzed waves, and $t(k)$ is the time required for computation with simultaneous analysis of $k$ waves.

The presented examples show that using the proposed method allows to speed-up the computation several times. Although in the absolute scale the difference between, e.g. 1 and 6 seconds seems small, it should be noted that the presented problem of light scattering calculations may be a part of a larger problem, in which the calculation of scattering should be performed repeatedly for different input data. Such a situation may occur, for example, in the inverse problem, where on the basis of the measured distri-
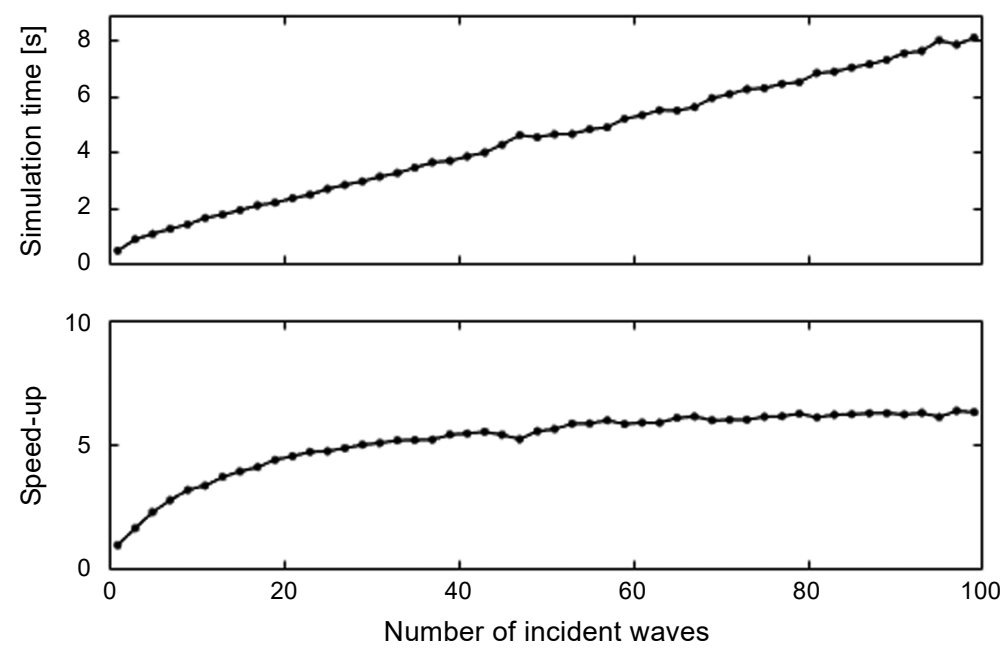

Fig. 2. Computational speed-up obtained by reusing computed trajectories in the MC algorithm to simulate the scattering of different incident electromagnetic waves. 
$\mathrm{T} \mathrm{a} \mathrm{b} 1 \mathrm{e}$ 4. Accuracy of Monte Carlo simulation of light scattering by the random conglomerate of particles.

\begin{tabular}{|c|c|c|c|c|c|c|}
\hline \multirow{2}{*}{$\begin{array}{l}\text { Number } \\
\text { of dipoles }\end{array}$} & \multirow{2}{*}{$\begin{array}{l}\text { Sphere } \\
\text { diameter }[\lambda]\end{array}$} & \multicolumn{2}{|c|}{ Monte Carlo simulation accuracy } & \multicolumn{3}{|c|}{ Jacobi iteration accuracy } \\
\hline & & $2 \times 10^{2}$ it. & $10^{3}$ it. & 1 it. & 2 it. & 3 it. \\
\hline 1000 & 0.25 & $2.65 \times 10^{-3}$ & $2.79 \times 10^{-3}$ & $2.45 \times 10^{-3}$ & $3.65 \times 10^{-3}$ & $5.08 \times 10^{-3}$ \\
\hline 2000 & 0.5 & $0.41 \times 10^{-3}$ & $0.30 \times 10^{-3}$ & $2.33 \times 10^{-3}$ & $0.07 \times 10^{-3}$ & $0.01 \times 10^{-3}$ \\
\hline 3000 & 0.75 & $0.56 \times 10^{-3}$ & $0.23 \times 10^{-3}$ & $3.60 \times 10^{-3}$ & $0.01 \times 10^{-3}$ & $0.00 \times 10^{-3}$ \\
\hline 4000 & 1.0 & $1.42 \times 10^{-3}$ & $0.52 \times 10^{-3}$ & $4.92 \times 10^{-3}$ & $0.10 \times 10^{-3}$ & $0.02 \times 10^{-3}$ \\
\hline 5000 & 0.25 & $0.34 \times 10^{-3}$ & $0.49 \times 10^{-3}$ & $5.93 \times 10^{-3}$ & $0.05 \times 10^{-3}$ & $0.01 \times 10^{-3}$ \\
\hline
\end{tabular}

$\mathrm{T} \mathrm{a} \mathrm{b} 1$ e 5. Time of Monte Carlo simulation of light scattering by the random conglomerate of particles.

\begin{tabular}{llccccc}
\hline $\begin{array}{l}\text { Number } \\
\text { of dipoles }\end{array}$ & $\begin{array}{l}\text { Sphere } \\
\text { diameter }[\lambda]\end{array}$ & \multicolumn{2}{c}{ Monte Carlo simulation time $[\mathrm{ms}]$} & \multicolumn{3}{c}{ Jacobi iteration time [ms] } \\
\cline { 3 - 7 } & $2 \times 10^{2}$ it. & $10^{3}$ it. & 1 it. & 2 it. & 3 it. \\
\hline 1000 & 0.25 & 48.0 & 161.2 & 0.074 & 10.2 & 15.8 \\
2000 & 0.5 & 66.2 & 323.5 & 0.123 & 29.0 & 58.4 \\
3000 & 0.75 & 104.0 & 503.1 & 0.011 & 64.2 & 125.4 \\
4000 & 1.0 & 155.8 & 816.7 & 0.004 & 110.9 & 190.1 \\
5000 & 0.25 & 221.2 & 1119.2 & 0.016 & 156.8 & 309.3 \\
\hline
\end{tabular}

bution of scattered radiation intensity resulting from different trajectories of individual scattering photons, the distribution and parameters of the scattering particles are determined. Such calculations are carried out using iterative methods, changing the parameters or the distribution of the scattering particles in each iteration. With many particles, the number of needed iterations can reach tens of thousands or more, which requires dozens of hours of calculation. In this case, shortening the calculation time several times can lead to the significant saving of time.

\subsection{Scattering by a random conglomerate}

The same numerical procedure as described in Section 3.1 was applied to compute the scattering cross-section of a random conglomerate of particles with a diameter equal to $0.2 \lambda$. The volume of the conglomerate was equal to $(10 \times 10 \times 200) \lambda^{3}$. The number of particles inside the volume was varying from 1000 to 5000. Each particle was represented by a single dipole. Obtained accuracies and computation times are presented in Tables 4 and 5.

\section{Discussion and conclusions}

The presented research shows that for a typical light scattering problem treated by the VIE and DDA methods, i.e. scattering of the light by a particle with a size comparable with the light wavelength, the $\mathrm{MC}$ algorithm is of a limited use. First, the direct treatment of the underlying system of equations with the $\mathrm{MC}$ algorithm cannot be done due to the problems with convergence. However, we showed that this problem can be easily solved using the proper preconditioning technique. With such preconditioning, the 
$\mathrm{MC}$ algorithm is convergent and leads to satisfactory results even if the length of the trajectories is limited to a small number. However, the efficiency of this method is inferior comparing to the Jacobi iteration scheme. Therefore, even if one needs to perform a fast, approximate solution to the analyzed scattering problem, it is more efficient to use the Jacobi iteration.

There are two rules of thumbs that concern the DDA computations: $i$ ) the distance between the two dipoles should be smaller than 0.1 of wavelength inside the scattering particle and $i i$ ) the number of dipoles along the smallest dimension of the particle should be greater than 10 [22]. Since the goal of this study was to compare two algorithms for solving the linear system, these rules were not met in a part of the presented simulations. However, the data presented in Table 1 show that meeting these requirements may be difficult if one wants to achieve the convergence of the $\mathrm{MC}$ algorithm. Also, one should notice that the above-mentioned rules of thumb were developed for large scattering particles and not sparsely distributed random media.

In the presented study, we considered only non-absorbing particles. However, the application of the MC algorithm for particles with the nonzero imaginary part of the refractive index is straightforward. This simplicity of dealing with absorbing particles is characteristic for the entire group of numerical methods that solve Maxwell's equations in the frequency domain.

The efficiency of the MC algorithm can be improved by various methods. For example, the so-called importance sampling technique [13], in which the trajectories with higher impacts are generated with higher probabilities might be applied. Also, the $\mathrm{MC}$ algorithm can be very easily parallelized. However, there are efficient algorithms for parallelizing matrix multiplication that is the main part of the Jacobi iterations. Also, such multiplication can be speed-up using the fast Fourier transform (FFT) algorithm. The comparison of highly optimized versions of both the MC and Jacobi iterations should be performed and reported in future studies. However, it is unlikely that the difference exceeding one order of magnitude shown in the results presented in this paper will be evened just by the optimization of the numerical method.

The results presented in Section 3.2 show that the efficiency of the MC algorithm can be increased if the numerical analysis needs to be done with not one but many different incident electromagnetic fields. This included changes in the polarization, the propagation direction, and the shape (e.g. the Gaussian beam with various parameters). The former two cases are especially common in the numerical analysis of light scattering. In the presented research, the reduction of computation time up to six times was achieved. This number is likely to increase in the case of longer trajectories when the larger part of the computations can be reused.

The comparison of the MC algorithm and the Jacobi iteration leads to different conclusions in the case of light scattering by conglomerates of randomly distributed particles. The results presented in Section 3.3 show that using only 200 trajectories in the case of over 1000 particles can improve the relative error of the result comparing to the approximation of the total electric field with the incident electric field. Still, the needed computation time was slightly higher than that for the two iterations of the Jacobi scheme, 
which leads to better results; but if one considers the computation speed-up presented in Fig. 2, the performance of the MC algorithm can be better.

The arguable issue is whether the decreasing of the relative error by including higher -order terms of Neumann series is required if the approximation of the total field by the incident fields leads to the relative error lower than $1 \%$. This is certainly the satisfactory accuracy in many applications but there are known physical phenomena that are attributed to the multiple scattering of light which is represented by the higher order terms of Neumann expansions. An example of such a phenomenon is the coherent-backscattering effect that increases the amplitude of the wave scattered at the angle of $180^{\circ}[23,24]$. Numerical analysis of such phenomena requires better approximation of the scattered waves and for that the MC algorithm may be used.

The main conclusions from the presented research are: $i$ ) with proper conditioning, the $\mathrm{MC}$ algorithm can be used to solve discretized VIE for light scattering problems in the cases of both large particles and random conglomerates of small particles, $i i$ ) the efficiency of the MC algorithm can be increased several times by simultaneous analysis of different incident electromagnetic waves, iii) it is unlikely that the MC algorithm will be competitive with other approaches to analyzing light scattering by the single large particles, and $i v$ ) it is possible that the $\mathrm{MC}$ algorithm might be useful for analysis of light scattering by random conglomerates of small scattering particles.

Acknowledgments - This research work has been supported by The National Science Centre, Poland under grant No. 2014/15/N/ST7/04903 and DS program of Faculty of Electronics, Telecommunications and Informatics, Gdańsk University of Technology.

\section{References}

[1] Yurkin M.A., Hoekstra A.G., The discrete dipole approximation: an overview and recent developments, Journal of Quantitative Spectroscopy and Radiative Transfer 106(1-3), 2007, pp. 558-589, DOI: 10.1016/j.jqsrt.2007.01.034.

[2] Mishchenko M.I., Electromagnetic Scattering by Particles and Particle Groups: An Introduction, Cambridge University Press, Cambridge, 2014.

[3] Draine B.T., Flatau P.J., Discrete-dipole approximation for scattering calculations, Journal of the Optical Society of America A 11(4), 1994, pp. 1491-1499, DOI: 10.1364/JOSAA.11.001491.

[4] Mischchenko M.I., Travis L.D., Mackowski D.W., T-matrix computations of light scattering by nonspherical particles: a review, Journal of Quantitative Spectroscopy and Radiative Transfer 55(5), 1996, pp. 535-575, DOI: 10.1016/0022-4073(96)00002-7.

[5] Mackowski D.W., Mishchenko M.I., A multiple sphere T-matrix Fortran code for use on parallel computer clusters, Journal of Quantitative Spectroscopy and Radiative Transfer 112(13), 2011, pp. 2182 -2192, DOI: 10.1016/j.jqsrt.2011.02.019.

[6] Martelli G.Z.F., Del Bainco S., Ismaelli A., Light Propagation Through Biological Tissue and Other Diffusive Media: Theory, Solutions and Software, SPIE Press Monograph, Vol. PM193, Bellingham, 2010.

[7] Wang L., JACQues S.L., Zheng L., MCML - Monte Carlo modeling of light transport in multi-layered tissues, Computer Methods and Programs in Biomedicine 47(2), 1995, pp. 131-146, DOI: $\underline{10.1016 /}$ 0169-2607(95)01640-F.

[8] JIN J-M., The Finite Element Method in Electromagnetics, 3rd Ed., Wiley, New York, 2014. 
[9] Taflove A., Hagness S.C., Computational Electrodynamics: The Finite-Difference Time-Domain Method, 3rd Ed., Artech House, Norwood, MA, 2005.

[10] Van De Hulst H.C., Light Scattering by Small Particles, Dover Publications, New York, 1981.

[11] Devaney A.J., Mathematical Foundations of Imaging, Tomography and Wavefield Inversion, Cambridge University Press, Cambridge, 2012.

[12] Goodman J.J., Draine B.T., Flatau P.J., Application offast-Fourier-transform techniques to the discrete-dipole approximation, Optics Letters 16(15), 1991, pp. 1198-1200, DOI: 10.1364/OL.16.001198.

[13] Dunn W.L., Shultis J.K., Exploring Monte Carlo Methods, Elsevier Science, Amsterdam, 2011.

[14] Doucet A., Johansen A.M., TAdić V.B., On solving integral equations using Markov chain Monte Carlo methods, Applied Mathematics and Computation 216(10), 2010, pp. 2869-2880, DOI: $10.1016 /$ j.amc.2010.03.138.

[15] Ji H., Mascagni M., Li Y., Convergence analysis of Markov chain Monte Carlo linear solvers using Ulam-von Neumann algorithm, Computer Science Faculty Publications 51(4), 2013, pp. 2107-2122, DOI: $10.1137 / 130904867$.

[16] SRinivasan A., Monte Carlo linear solvers with non-diagonal splitting, Mathematics and Computers in Simulation 80(6), 2010, pp. 1133-1143, DOI: 10.1016/j.matcom.2009.03.010.

[17] Pereira P.F., Sherif S.S., Simulation of the interaction of light and tissue in a large volume using a Markov chain Monte Carlo method, Proceedings of SPIE 8412, 2012, article 841218, DOI: 10.1117 I 12.2001455 .

[18] Kraszewsi M., Pluciński J., Coherent-wave Monte Carlo method for simulating light propagation in tissue, Proceedings of SPIE 9706, 2016, article 970611, DOI: 10.1117/12.2213213.

[19] LaKhtAKIA A., Strong and weak forms of the method of moments and the coupled dipole method for scattering of time-harmonic electromagnetic fields, International Journal of Modern Physics C 3(3), 1992, pp. 583-603, DOI: 10.1142/S0129183192000385.

[20] SAAD Y., Iterative methods for sparse linear systems, 2nd Ed., Society for Industrial and Applied Mathematics, Philadelphia, 2003.

[21] Loke V.L.Y., Mengüç M.P., Nieminem T.A., Discrete-dipole approximation with surface interaction: computational toolbox for MATLAB, Journal of Quantitative Spectroscopy and Radiative Transfer 112(11), 2011, pp. 1711-1725, DOI: 10.1016/j.jqsrt.2011.03.012.

[22] SKORUPSKI K., Using the DDA (discrete dipole approximation) method in determining the extinction cross section of black carbon, Metrology and Measurement Systems 22(1), 2015, pp. 153-164, DOI: $10.1515 / \mathrm{mms}-2015-0013$.

[23] Van Rossum M.C.W., Nieuwenhuizen Th.M., Multiple scattering of classical waves: microscopy, mesoscopy and diffusion, Reviews of Modern Physics 71(1), 1999, pp. 313-371, DOI: $\underline{10.1103 / \mathrm{Rev}}$ ModPhys.71.313.

[24] Doronin A., Radosevich A.J., Backman V., Meglinski I., Two electric field Monte Carlo models of coherent backscattering of polarized light, Journal of the Optical Society of America A 31(11), 2014, pp. 2394-2400, DOI: 10.1364/JOSAA.31.002394. 